[9] T. Mori and A. Derese, "A brief summary of the bounds on the solution of the algebraic matrix equations in control theory," Int. J. Control, vol. 39, pp. 247-256, 1984.

[10] T. Mori and H. Kokame, "Stability margin estimation of real Schur polynomials via established stability tests," Trans. IEICE Fundament., vol. E81-A, pp. 1301-1304, 1998.

[11] _ "A note on the stability of delta-operator-induced systems," IEEE Trans. Automat. Contr., vol. 45, pp. 1885-1886, Oct. 2000.

[12] M. Mrabti and A. Hmamed, "Bounds for the solution of the Lyapunov matrix equation-A unified approach," Syst. Control Lett., vol. 18, pp. 73-81, 1992.

[13] C. H. Lee, "Upper and lower matrix bounds of the solution for the discrete Lyapunov equation," IEEE Trans. Automat. Contr., vol. 41, pp. 1338-1341, Sept. 1996

[14] — "On the matrix bounds for the solution matrix of the discrete algebraic Riccati equation," IEEE Trans. Circuits Syst. I, vol. 43, pp. 402-407, May 1996.

[15] M. K. Tippert and D. Marchesin, "Upper bounds for the solution of the discrete algebraic Lyapunov equation," Automatica, vol. 35, pp. 1485-1489, 1999.

\section{Optimal Tracking Performance for SIMO Systems}

\author{
Gang Chen, Jie Chen, and Rick Middleton
}

\begin{abstract}
This note studies the optimal tracking problem for linear time-invariant single-input-multiple-output systems responding to a step reference signal. An integral square error criterion is used as the measure for tracking performance. Explicit expressions are developed for the optimal tracking error. These results characterize how nonsquare plants may pose additional difficulties for tracking, other than those resulted from nonminimum phase zeros and unstable poles.
\end{abstract}

Index Terms-Optimal tracking, performance limitation, singleinput-multiple-output (SIMO) systems.

\section{INTRODUCTION}

This note studies the optimal tracking performance for linear timeinvariant single-input-multiple-output (SIMO) systems responding to a step reference signal. The cost function employed is the integral of the squared norm of tracking error, which is to be optimized over all possible stabilizing controllers. Optimal tracking problem is a well-studied topic for single-input-single-output (SISO) systems [9], and for right-invertible multiple-input-multiple-output (MIMO) systems [3], [11], [12]. The existing results show that, in general, the unstable poles and nonminimum phase zeros of the plant impose inevitable limitations on tracking performance. More recently, there has also been effort to extend these results to nonsquare systems, which, to date, has been largely concentrated on single-input-two-output (SITO) systems. For example, Freudenberg and Middleton [7], [8]

Manuscript received February 6, 2002; revised April 15, 2002. Recommended by Associate Editor T. Iwasaki. This work was supported by the National Science Foundation.

G. Chen and J. Chen are with the Department of Electrical Engineering, University of California, Riverside, CA USA 92521-0425 (e-mail: gchen@ee.ucr.edu; jchen@ee.ucr.edu).

R. Middleton is with the Department of Electrical and Computer Engineering, The University of Newcastle, Newcastle, NSW 2308, Australia (e-mail: rick@ee.newcastle.edu.au).

Digital Object Identifier 10.1109/TAC.2002.803559. considered "algebraic" design tradeoffs between feedback properties examined at different loop-breaking points, in which the analysis is focused on a single frequency. Woodyatt [15] studied Bode integral relations and an optimal tracking problem for SITO systems. For general nonright-invertible systems, Woodyatt et al. [14] discussed the problem of cheap control performance.

A performance study of nonright-invertible systems bears both practical and theoretical relevance. While in the majority of multivariable control research the plants are assumed to be square, many systems are not [10]. Furthermore, some square MIMO systems [6], [8] may exhibit a behavior of nonsquare systems, due to almost rank deficiency resulted from actuator faults and constraints and, thus, are essentially of a nonright-invertible nature. Intuitively, the nonsquare structure brings about a distinctively new class of constraints. Indeed, when the optimal tracking problem is considered for a nonright-invertible system, the fact that fewer inputs are available to control more outputs will make the control of such a system more difficult. Our primary interest in this note is to characterize analytically how tracking performance may be fundamentally constrained by plant structure. In turn, the understanding of this inherent design difficulty will provide rules of thumb and benchmarks for control designers and thus aid in feedback design.

This note extends [3], [12], and [15]. We first develop a general formula for the minimal tracking error, which is expressed in terms of the inner factor of the plant, and is valid for both right-invertible and nonright-invertible systems. Based on this result, we then derive explicit expressions for the minimal tracking error concerning SIMO systems. These results indicate that the achievable tracking performance is constrained not only by the nonminimum phase zeros but also by the total variation of the plant direction with frequency; the latter effect is quantified by a weighted integral of the relative angle between the plant direction at the frequencies $\omega$ and 0 . Indeed, it turns out that a rapid change of plant direction at low frequencies will impose a more severe constraint upon the achievable tracking performance. Furthermore, for unstable SIMO plants, unlike in the case of right-invertible systems, it will also be shown that the unstable poles may still affect the tracking performance despite the fact that the plant may be minimum phase.

The rest of this note is organized as follows. In Section II, we describe the setup of unity feedback systems and introduce the relevant mathematical background. Section III presents our main results. An illustrative example is presented in Section IV. Section V concludes the discussion.

Some of the results in this note were presented previously in [1].

\section{PRELIMINARIES}

The notation used in this note is collected in the following. We denote the complex plane by $\mathbb{C}$ and open right half plane by $\mathbb{C}_{+}$. For a complex number $s$, its conjugate is denoted by $\bar{s}$ and its real part by $\operatorname{Re}(s)$. Given a matrix $M \in \mathbb{C}^{m \times n}$, denote its transpose by $M^{T}$, its conjugate transpose by $M^{H}$, and its range (i.e., column space) by $\mathcal{R}(M)$. The Euclidean vector norm of a vector $\nu$ is $\|\nu\|=\sqrt{\nu^{H} \nu}$. The angle between the directions of two vectors $a, b \in \mathbb{C}^{n}$ is defined as $\angle(a, b)=\arccos \left(\left|a^{H} b\right| /(\|a\|\|b\|)\right)$. Given a matrix function $f(s) \in \mathbb{C}^{m \times n}$, let $f^{\sim}(s)=f^{T}(-s)$. For any signal $u(t)$, denote its Laplace transform by $\hat{u}(s)$. Throughout this note, the vectors and matrices are assumed to have compatible dimensions, and the dimensions are thus omitted for simplicity. The space $\mathcal{L}_{2}$ is the Hilbert space with inner product

$$
\langle f, g\rangle:=\frac{1}{2 \pi} \int_{-\infty}^{\infty} f^{H}(j \omega) g(j \omega) d \omega
$$




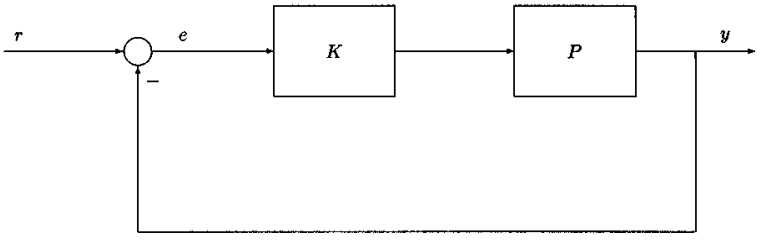

Fig. 1. The unity feedback tracking system.

which further induces the $\mathcal{L}_{2}$ norm $\|f\|_{2}:=\sqrt{\langle f, f\rangle}$. For any $f, g \in$ $\mathcal{L}_{2}$, they are orthogonal if $\langle f, g\rangle=0$. It is well known that $\mathcal{L}_{2}$ can be decomposed into two orthogonal subspaces $\mathcal{H}_{2}$ and $\mathcal{H}_{2}^{\perp}$ given by [5]

$$
\begin{aligned}
\mathcal{H}_{2} & :=\left\{f: f(s) \text { analytic in } \mathbb{C}_{+},\right. \\
\|f\|_{2}^{2} . & \left.:=\sup _{\sigma>0} \frac{1}{2 \pi} \int_{-\infty}^{\infty}\|f(\sigma+j \omega)\|^{2} d \omega<\infty\right\}
\end{aligned}
$$

and

$$
\begin{aligned}
\mathcal{H}_{2}^{\perp} & :=\left\{f: f(s) \text { analytic in } \mathbb{C}_{-},\right. \\
\|f\|_{2}^{2} & \left.:=\sup _{\sigma<0} \frac{1}{2 \pi} \int_{-\infty}^{\infty}\|f(\sigma+j \omega)\|^{2} d \omega<\infty\right\} .
\end{aligned}
$$

Finally, we denote the set of all stable, proper, rational transfer function matrices by $\mathbb{R} \mathcal{H}_{\infty}$.

We consider the standard unity feedback, one-parameter control scheme depicted in Fig. 1; our subsequent development, however, extends readily to two-parameter control systems. In this configuration, $P$ denotes the plant model and $K$ the compensator, with transfer function matrices of $P(s)$ and $K(s)$, respectively. Hereafter, whenever convenient, we omit the dependence of the transfer functions on the frequency variable $s$. We shall assume throughout that the feedback system is stable, which implies that $P$ and $K$ have no hidden modes in the closed right half plane. In Fig. 1, the signals $r, e$, and $y$ represent the command input, the error, and the system output, respectively. Moreover, define the output open-loop transfer function by $L:=P K$, and the output sensitivity function by $S:=(I+L)^{-1}$.

For the rational transfer function matrix $P$, let its right and left coprime factorization be given by

$$
P=N M^{-1}=\tilde{M}^{-1} \tilde{N}
$$

where $N, M, \tilde{N}, \tilde{M} \in \mathbb{R} \mathcal{H}_{\infty}$ and satisfy the double Bezout identity

$$
\left[\begin{array}{rr}
\tilde{X} & -\tilde{Y} \\
-\tilde{N} & \tilde{M}
\end{array}\right]\left[\begin{array}{cc}
M & Y \\
N & X
\end{array}\right]=I
$$

for some $X, Y, \tilde{X}, \tilde{Y} \in \mathbb{R} \mathcal{H}_{\infty}$. It is well known that to stabilize $P$ every compensator $K$ is characterized by the Youla parameterization [5]

$$
\begin{aligned}
\mathcal{K}:=\{K: K= & -(Y-M Q)(X-N Q)^{-1} \\
& \left.=-(\tilde{X}-Q \tilde{N})^{-1}(\tilde{Y}-Q \tilde{M}), Q \in \mathbb{R} \mathcal{H}_{\infty}\right\} .
\end{aligned}
$$

In particular, when $P$ is stable, then we can select $N=\tilde{N}=P$, $\tilde{X}=M=I, X=\tilde{M}=I$, and $Y=\tilde{Y}=0$. As a result, the parameterization $(3)$ reduces to

$$
\mathcal{K}=\left\{K: K=Q(I-P Q)^{-1}=(I-Q P)^{-1} Q, Q \in \mathbb{R} \mathcal{H}_{\infty}\right\} .
$$

Furthermore, consider the function class

$$
\mathbb{F}:=\left\{f: f(s) \text { analytic in } \mathbb{C}_{+}, \lim _{R \rightarrow \infty} \max _{\theta \in[-(\pi / 2),(\pi / 2)]} \frac{\left|f\left(\operatorname{Re}^{j \theta}\right)\right|}{R}=0\right\} .
$$

The following integral formulas play an important role in our subsequent derivation. We remark that Lemma 2.1 can be found directly in [13] and Lemma 2.2 can be readily derived using the results in [4].
Lemma 2.1: Let $f(s) \in \mathbb{F}$ and denote $f(j \omega)=h(\omega)+j g(\omega)$. Suppose that $f(s)$ is conjugate symmetric, i.e., $f(s)=\overline{f(\bar{s})}$. Then

$$
f^{\prime}(0)=\lim _{\omega \rightarrow 0} \frac{g(\omega)}{\omega}=\frac{1}{\pi} \int_{-\infty}^{\infty} \frac{h(\omega)-h(0)}{\omega^{2}} d \omega .
$$

Lemma 2.2: Let $f(s)$ be a meromorphic function in $\mathbb{C}_{+}$. Suppose that $f(s)$ is conjugate symmetric and $\log f(s) \in \mathbb{F}$. Also, suppose that $f(s)$ has $N_{z}$ zeros $z_{i} \in \mathbb{C}_{+}, i=1, \ldots, N_{z}$, and $N_{p}$ poles $p_{i} \in \mathbb{C}_{+}$, $i=1, \ldots, N_{p}$, all counting multiplicities. Then, if $f(0) \neq 0$

$$
\int_{-\infty}^{\infty} \log \left|\frac{f(j \omega)}{f(0)}\right| \frac{d \omega}{\omega^{2}}=2 \pi \sum_{i=1}^{N_{z}} \frac{1}{z_{i}}-2 \pi \sum_{i=1}^{N_{p}} \frac{1}{p_{i}}+\pi \frac{f^{\prime}(0)}{f(0)} .
$$

Here, by a meromorphic function $f(s)$ in $\mathbb{C}_{+}$, we mean that $f(s)$ is analytic in $\mathbb{C}_{+}$except for poles [13].

\section{OPTIMAL TRACKING FOR SIMO SYSTEMS}

We consider a step reference signal of the form

$$
r(t)= \begin{cases}\nu, & t \geq 0 \\ 0, & t<0\end{cases}
$$

where $\nu=\left[\nu_{1}, \nu_{2}, \ldots, \nu_{n}\right]^{T} \in \mathbb{R}^{n}$ is a constant vector with $\|\nu\|=$ 1. Moreover, we assume that the system is initially at rest. For the given input signal $r$, the tracking performance of the system is defined as

$$
J:=\int_{0}^{\infty}\|e(t)\|^{2} d t
$$

Because $\hat{e}(s)=S(s) \hat{r}(s)$, it follows from the Parseval identity that

$$
J=\|S \hat{r}\|_{2}^{2}=\frac{1}{2 \pi} \int_{-\infty}^{\infty}\|S(j \omega) \hat{r}(j \omega)\|^{2} d \omega .
$$

The minimal tracking error achievable by all stabilizing compensators form the set $\mathcal{K}$ is defined by

$$
J^{*}:=\inf _{K \in \mathcal{K}} J
$$

Throughout this section, we make the following assumptions.

Assumption 3.1: $P(s)$ does not have transmission zero at $s=0$.

Assumption 3.2: For $r(t)$ defined in (7), $\nu \in \mathcal{R}(P(0))$.

We note that these assumptions are rather standard and general. To make the integral in (8) finite with the step reference signal (7), it is necessary that $S(s)$ has a transmission zero at $s=0$, with input zero direction $\nu$; here by a transmission zero $z$ of $S(s)$ with input direction $\nu$, we mean that $S(z) \nu=0$. Assumption 3.1 is then necessary to preclude any hidden pole-zero cancellation at $s=0$. Furthermore, Assumption 3.2 gives the condition of the step reference signal that a nonright-invertible system may track.

We proceed by first developing a general formula for $J^{*}$, which is valid for either right-invertible or nonright-invertible systems. Note that for such a stable plant $P$, it has an inner-outer factorization

$$
P=\Theta_{i} \Theta_{o}
$$

where $\Theta_{i}, \Theta_{o} \in \mathbb{R} \mathcal{H}_{\infty}$ are inner and outer factors, respectively. Note that $\tilde{\Theta_{i}}(s) \Theta_{i}(s)=I$ and $\Theta_{o}$ admits a right inverse in $\mathbb{R} \mathcal{H}_{\infty}$; see $[5$, p. 98] for more details.

Theorem 3.1: Let $r(t)$ be given by (7) and suppose that the plant $P$ is stable. Then

$$
J^{*}=-\nu^{H} \Theta_{i}^{\prime}(0) \Theta_{i}^{H}(0) \nu .
$$


Proof: Using the Youla parameterization (4), we get

$$
J^{*}=\inf _{Q \in \mathbb{R}^{\mathcal{H}}}\left\|(I-P Q) \frac{\nu}{s}\right\|_{2}^{2}
$$

in which $Q$ is selected such that $(I-P(0) Q(0)) \nu=0$ in order for $J^{*}$ to be finite. It is easy to verify that $\left[\begin{array}{c}\Theta_{i}^{-} \\ I-\Theta_{i}^{-} \Theta_{i}\end{array}\right]$ is an inner matrix. Hence

$$
\begin{aligned}
J^{*} & =\inf _{Q \in \mathbb{R} \mathcal{H}}\left\|\left[\begin{array}{c}
\Theta_{i} \\
I-\Theta_{i} \tilde{\Theta_{i}}
\end{array}\right](I-P Q) \frac{\nu}{s}\right\|_{2}^{2} \\
& =\inf _{Q \in \mathbb{R} \mathcal{H}_{\infty}}\left\|\left[\begin{array}{c}
\Theta_{i}-\Theta_{o} Q \\
I-\Theta_{i} \tilde{\Theta_{i}}
\end{array}\right] \frac{\nu}{s}\right\|_{2}^{2} \\
& =\inf _{Q \in \mathbb{R} \mathcal{H}_{\infty}}\left\|\left(\tilde{\Theta_{i}}-\Theta_{o} Q\right) \frac{\nu}{s}\right\|_{2}^{2}+\left\|\left(I-\Theta_{i} \tilde{\Theta_{i}}\right) \frac{\nu}{s}\right\|_{2}^{2} .
\end{aligned}
$$

Note that $\left(\tilde{\Theta_{i}}-\tilde{\Theta_{i}}(0)\right) \nu / s \in \mathcal{H}_{2}^{\perp}$. We may pick $Q \in \mathbb{R} \mathcal{H}_{\infty}$ such that $\tilde{\Theta_{i}}(0)-\Theta_{o}(0) Q(0)=0$, and hence $\left(\tilde{\Theta_{i}}(0)-\Theta_{o} Q\right) \nu / s \in \mathcal{H}_{2}$. As a result,

$$
\begin{aligned}
J^{*}=\inf _{Q \in \mathbb{R} \mathcal{H}_{\infty}} \|\left(\tilde{\Theta_{i}}(0)\right. & \left.-\Theta_{o} Q\right) \frac{\nu}{s} \|_{2}^{2} \\
& +\left\|\left(\tilde{\Theta_{i}}-\tilde{\Theta_{i}}(0)\right) \frac{\nu}{s}\right\|_{2}^{2}+\left\|\left(I-\Theta_{i} \tilde{\Theta_{i}}\right) \frac{\nu}{s}\right\|_{2}^{2} .
\end{aligned}
$$

Because $\Theta_{o}$ is outer, we have inf $\inf _{Q \in \mathbb{H}_{\infty}}\left\|\left(\tilde{\Theta_{i}}(0)-\Theta_{o} Q\right)(\nu / s)\right\|_{2}^{2}=$ 0 . A direct calculation then yields

$$
\begin{aligned}
J^{*} & =\left\|\left(\tilde{\Theta_{i}}-\tilde{\Theta_{i}}(0)\right) \frac{\nu}{s}\right\|_{2}^{2}+\left\|\left(I-\Theta_{i} \tilde{\Theta_{i}}\right) \frac{\nu}{s}\right\|_{2}^{2} \\
& =-\frac{1}{\pi} \int_{-\infty}^{\infty} \frac{\operatorname{Re}\left(\nu^{H} \Theta_{i}(j \omega) \Theta_{i}^{H}(0) \nu\right)-1}{\omega^{2}} d \omega .
\end{aligned}
$$

Define $f(s):=\nu^{H} \Theta_{i}(s) \Theta_{i}^{H}(0) \nu$. Under Assumption 3.2, we have $f(0)=1$. Invoking Lemma 2.1 then gives

$$
J^{*}=-f^{\prime}(0)=-\nu^{H} \Theta_{i}^{\prime}(0) \Theta_{i}^{H}(0) \nu .
$$

Remark 3.1: As shown in [3], [9], for SISO and right-invertible systems, the nonminimum phase zeros of the plant impose fundamental constraints on tracking performance. Since the inner part of the plant contains all the nonminimum phase zeros, it comes as no surprise that in (10) the inner factor $\Theta_{i}$ is involved. It is easy to show that for right-invertible systems Theorem 3.1 reduces to the known result in [3]. Indeed, for a right-invertible plant $P$, suppose that it has nonminimum phase zeros at $z_{i}, i=1, \ldots, N_{z}$. The inner part of $P$ can be formed as

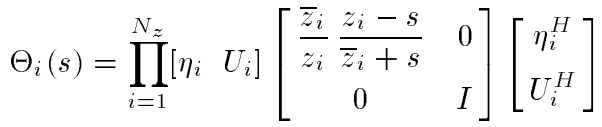

where the unitary vector $\eta_{i}$ can be iteratively computed from the zero direction vectors of $P$, and $U_{i}$ is a matrix whose columns, together with $\eta_{i}$, form an orthonormal basis of the corresponding Euclidean space; we refer the reader to [2] for this iterative procedure. Therefore, invoking (10) yields

$$
\begin{aligned}
& J^{*}=-\sum_{i=1}^{N_{z}} \nu^{H}\left[\eta_{i} U_{i}\right]\left[\begin{array}{cc}
-\frac{2 \operatorname{Re}\left(z_{i}\right)}{\left|z_{i}\right|^{2}} & 0 \\
0 & 0
\end{array}\right]\left[\begin{array}{c}
\eta_{i}^{H} \\
U_{i}^{H}
\end{array}\right] \nu \\
& =\sum_{i=1}^{N_{z}} \frac{2 \operatorname{Re}\left(z_{i}\right)}{\left|z_{i}\right|^{2}}\left|\eta_{i}^{H} \nu\right|^{2} \text {. }
\end{aligned}
$$

More generally, Theorem 3.1 is applicable to plants that need not be right invertible and, hence, lays the very foundation in our subsequent study of SIMO systems.

\section{A. Stable SIMO Plants}

We now derive an analytical expression of the minimal tracking error $J^{*}$ for SIMO systems. We consider first stable plants, and subsequently extend the results to unstable plants in Section III-B. Let $P$ and $K$ be

$$
\begin{aligned}
P(s) & =\left[P_{1}(s), P_{2}(s), \ldots, P_{n}(s)\right]^{T} \\
K(s) & =\left[K_{1}(s), K_{2}(s), \ldots, K_{n}(s)\right]
\end{aligned}
$$

where $P_{i}(s)$ and $K_{i}(s), 1 \leq i \leq n$, are scalar transfer functions.

Theorem 3.2: Let $r(t)$ be given by (7), and suppose that the SIMO plant $P$ is stable. Also, suppose that $P_{i}(s)$ has nonminimum phase zeros at $z_{i k}, k=1, \ldots, N_{i}$. Then

$$
\begin{aligned}
J^{*}=\sum_{i \in \mathcal{I}} \nu_{i}^{2} & \sum_{k=1}^{N_{i}} \frac{2 \operatorname{Re}\left(z_{i k}\right)}{\left|z_{i k}\right|^{2}} \\
& +\frac{1}{\pi} \sum_{i \in \mathcal{I}} \nu_{i}^{2} \int_{0}^{\infty} \log \left(\frac{\left|P_{i}(0)\right|^{2}}{\|P(0)\|^{2}} \frac{\|P(j \omega)\|^{2}}{\left|P_{i}(j \omega)\right|^{2}}\right) \frac{d \omega}{\omega^{2}}
\end{aligned}
$$

where $\mathcal{I}$ is the index set defined by $\mathcal{I}:=\left\{i: P_{i}(0) \neq 0\right\}$.

Proof: The stable SIMO plant $P$ has an inner-outer factorization $\Theta_{i} \Theta_{o}$. Denote the inner factor $\Theta_{i}$ as

$$
\Theta_{i}(s)=\left[\theta_{1}(s), \theta_{2}(s), \ldots, \theta_{n}(s)\right]^{T} .
$$

Since $\nu \in \mathcal{R}(P(0)), \nu=c P(0)=c \Theta_{i}(0) \Theta_{o}(0)$ for some $c \in \mathbb{R}$. Furthermore, since $\|\nu\|^{2}=\left|c \Theta_{o}(0)\right|^{2}=1$, we may take, with no loss of generality, $\nu=\Theta_{i}(0)$. Plugging this $\nu$ into (10) yields

$$
J^{*}=-\Theta_{i}^{H}(0) \Theta_{i}^{\prime}(0)=-\sum_{i \in \mathcal{I}} \nu_{i}^{2} \frac{\theta_{i}^{\prime}(0)}{\theta_{i}(0)} .
$$

Because $\theta_{i}(s)$ has the same set of nonminimum phase zeros as $P_{i}(s)$, by invoking Lemma 2.2 we obtain

$$
\frac{\theta_{i}^{\prime}(0)}{\theta_{i}(0)}=-2 \sum_{k=1}^{N_{i}} \frac{1}{z_{i k}}+\frac{1}{\pi} \int_{-\infty}^{\infty} \log \left|\frac{\theta_{i}(j \omega)}{\theta_{i}(0)}\right| \frac{d \omega}{\omega^{2}} .
$$

The result (11) then follows by noting that $\left|\theta_{i}(j \omega)\right|=$ $\left|P_{i}(j \omega)\right| /\|P(j \omega)\|$.

The expression of the optimal tracking error for SIMO systems shares some similarities with its counterparts for SISO and right-invertible MIMO systems, in that the nonminimum phase zeros of the plant are also seen to constrain the achievable tracking performance. However, it is interesting to note that the zeros of $P_{i}(s)$ have an effect dependent on $\nu_{i}$. Indeed, since the reference signal is confined in $\mathcal{R}(P(0))$, the first term on the right-hand side of (11) may still be zero if $P_{i}(s)$ does not have nonminimum phase zeros for $i \in \mathcal{I}$. The extra integral term on the right-hand side of (11) points to the main difference between the right-invertible MIMO and SIMO systems. We will see shortly that this second term, which is nonnegative, accounts for the effect brought about by changes in the plant direction with frequency. This can be attributed to the deficiency in the freedom of control.

We next illustrate Theorem 3.2 by considering a special case, where the plant $P$ satisfies

$$
P(0)=\left[P_{1}(0), 0, \ldots, 0\right]^{T} .
$$

The following corollary is immediate from Theorem 3.2. 
Corollary 3.1: Let $r(t)$ be given by (7), and suppose that the SIMO plant $P$ is stable and satisfies (12). Also, suppose that $P_{1}(s)$ has nonminimum phase zeros at $z_{k}, k=1, \ldots, N_{1}$. Then

$$
J^{*}=\sum_{k=1}^{N_{1}} \frac{2 \operatorname{Re}\left(z_{k}\right)}{\left|z_{k}\right|^{2}}+\frac{1}{\pi} \int_{0}^{\infty} \log \left(\frac{\|P(j \omega)\|^{2}}{\left|P_{1}(j \omega)\right|^{2}}\right) \frac{d \omega}{\omega^{2}} .
$$

Remark 3.2: Corollary 3.1 illustrates in a more explicit manner the nature of the tracking performance limitation encountered in SIMO systems. Note that the second term on the right side of (13) is nonnegative, and is zero if and only if

$$
P_{i}(j \omega) \equiv 0, \quad 2 \leq i \leq n .
$$

Under this circumstance, the plant does not change its direction with frequency. Rewrite (13) as

$$
J^{*}=\sum_{k=1}^{N_{1}} \frac{2 \operatorname{Re}\left(z_{k}\right)}{\left|z_{k}\right|^{2}}+\frac{1}{\pi} \int_{0}^{\infty} \log \left(\frac{1}{\cos ^{2} \phi(\omega)}\right) \frac{d \omega}{\omega^{2}}
$$

where $\phi(\omega)$ is the angle between the plant directions at the frequencies 0 and $\omega$ with

$$
\cos \phi(\omega)=\frac{\left|\sum_{i=1}^{n} P_{i}(0) P_{i}(j \omega)\right|}{\|P(0)\|\|P(j \omega)\|}=\frac{\left|P_{1}(j \omega)\right|}{\|P(j \omega)\|} .
$$

In this sense, the second term in (13) describes the total variation of the plant direction in the whole frequency range. This observation extends to general SIMO plants. Indeed, for any given SIMO plant $P$, we may construct the projection matrix

$$
\Lambda:=\left[\frac{P(0)}{\|P(0)\|}, U\right]
$$

where $P(0) /\|P(0)\|$ and the columns of $U$ form an orthonormal basis of $\mathbb{R}^{n}$. Let $P^{*}=\Lambda^{H} P$. Then $P^{*}$ possesses the structure of (12), to which the above remarks apply. It is straightforward to verify that the performance for $P$ to track $\nu$ is the same as that for $P^{*}$ to track $\Lambda^{H} \nu$, while the latter performance depends on the direction change of $P^{*}(j \omega)$ with frequency. Since the projection preserves the relevant angles, i.e.,

$$
\angle(P(0), P(j \omega))=\angle\left(P^{*}(0), P^{*}(j \omega)\right)
$$

it follows that the performance for $P$ to track $\nu$ depends on the direction change of $P(j \omega)$ in general.

Furthermore, since the weighting function $1 / \omega^{2}$ decays fast as $\omega$ increases, Corollary 3.1 implies that a rapid change of plant direction at low frequencies will have a more detrimental effect on the achievable tracking performance. This observation is reinforced by the following corollaries.

Corollary 3.2: Assume that for some constants $c, \omega_{0}>0$

$$
\frac{\|P(j \omega)\|^{2}}{\left|P_{1}(j \omega)\right|^{2}} \geq 1+\frac{c \omega^{2}}{\omega^{2}+\omega_{0}^{2}}, \quad \omega \in\left[0, \omega_{0}\right] .
$$

Then, under the assumptions in Corollary 3.1

$$
J^{*} \geq \sum_{k=1}^{N_{1}} \frac{2 \operatorname{Re}\left(z_{k}\right)}{\left|z_{k}\right|^{2}}+\frac{1}{2 \omega_{0}} \log \left(1+\frac{c}{2}\right) .
$$

Proof: With the inequality

$$
\log (1+c x) \geq 2 \log \left(1+\frac{c}{2}\right) x, \quad x \in\left[0, \frac{1}{2}\right]
$$

we complete the proof by obtaining

$$
\begin{aligned}
J^{*} & \geq \sum_{k=1}^{N_{1}} \frac{2 \operatorname{Re}\left(z_{k}\right)}{\left|z_{k}\right|^{2}}+\frac{1}{\pi} \int_{0}^{\omega_{0}} \log \left(1+\frac{c \omega^{2}}{\omega^{2}+\omega_{0}^{2}}\right) \frac{d \omega}{\omega^{2}} \\
& \geq \sum_{k=1}^{N_{1}} \frac{2 \operatorname{Re}\left(z_{k}\right)}{\left|z_{k}\right|^{2}}+\frac{2 \log \left(1+\frac{c}{2}\right)}{\pi} \int_{0}^{\omega_{0}} \frac{1}{\omega^{2}+\omega_{0}^{2}} d \omega .
\end{aligned}
$$

Corollary 3.3: Assume that for some constants $\delta, k, \omega_{c}>0$

$$
\frac{\|P(j \omega)\|^{2}}{\left|P_{1}(j \omega)\right|^{2}} \geq \delta\left(\frac{\omega}{\omega_{c}}\right)^{k}, \quad \omega \in\left[\omega_{c}, \infty\right] .
$$

Then, under the assumptions in Corollary 3.1

$$
J^{*} \geq \sum_{k=1}^{N_{1}} \frac{2 \operatorname{Re}\left(z_{k}\right)}{\left|z_{k}\right|^{2}}+\frac{1}{\pi \omega_{c}}(\log \delta+k) .
$$

Proof: In light of (16), the proof is completed by weakening $J^{*}$

$$
\begin{aligned}
J^{*} & \geq \sum_{k=1}^{N_{1}} \frac{2 \operatorname{Re}\left(z_{k}\right)}{\left|z_{k}\right|^{2}}+\frac{1}{\pi} \int_{\omega_{c}}^{\infty} \log \left(\delta\left(\frac{\omega}{\omega_{c}}\right)^{k}\right) \frac{d \omega}{\omega^{2}} \\
& =\sum_{k=1}^{N_{1}} \frac{2 \operatorname{Re}\left(z_{k}\right)}{\left|z_{k}\right|^{2}}+\frac{1}{\pi \omega_{c}}(\log \delta+k) .
\end{aligned}
$$

\section{B. Unstable SIMO Plants}

More generally, by using the technique developed in [3], we further extend the preceding results to unstable SIMO plants. Suppose that $P(s)$ has simple unstable poles at $p_{i}, i=1, \ldots, N_{p}$ and factorize $P(s)$ as

$$
P(s)=P_{s}(s) B^{-1}(s)=\Theta_{i}(s) \Theta_{o}(s) B^{-1}(s)
$$

where $P_{s}$ is stable, $B(s):=\prod_{i=1}^{N_{p}}\left(\left(s-p_{i}\right) /\left(s+\bar{p}_{i}\right)\right)$, and $\Theta_{i}, \Theta_{o}$ are the inner and outer factors of $P_{s}$.

Theorem 3.3: Let $r(t)$ be given by (7) and $P(s)$ by (18). Then

$$
J^{*}=J_{s}^{*}+J_{u}^{*}
$$

where $J_{s}^{*}$ is the optimal tracking error corresponding to $P_{s}$, and

$$
\begin{aligned}
J_{u}^{*}=\sum_{i, j \in \mathcal{J}} \frac{4 \operatorname{Re}\left(p_{i}\right) \operatorname{Re}\left(p_{j}\right)}{\left(\bar{p}_{i}+p_{j}\right) p_{i} \bar{p}_{j} \bar{\alpha}_{i} \alpha_{j}}\left(1-\tilde{\Theta_{i}}\left(\bar{p}_{i}\right) \Theta_{i}(0)\right) \\
\cdot\left(1-\tilde{\Theta_{i}}\left(p_{j}\right) \Theta_{i}(0)\right)
\end{aligned}
$$

with $\alpha_{i}:=\prod_{j \in \mathcal{J}, j \neq i}\left(\bar{p}_{j} / p_{j}\right)\left(\left(p_{j}-p_{i}\right) /\left(\bar{p}_{j}+p_{i}\right)\right)$, where $\mathcal{J}$ is the index set defined by $\mathcal{J}:=\left\{i: \tilde{M}\left(p_{i}\right) \nu=0\right\}$.

Proof: Using Youla parameterization (3) yields $S=$ $(X-N Q) \tilde{M}$. Hence

$$
J^{*}=\inf _{Q \in \mathbb{R} \mathcal{H}_{\infty}}\left\|(X \tilde{M}-N Q \tilde{M}) \frac{\nu}{s}\right\|_{2}^{2} .
$$

It is easy to verify that

$$
X \tilde{M}=I+P Y \tilde{M}=I+\Theta_{i} \Theta_{o} B^{-1} Y \tilde{M}, \quad N=\Theta_{i} C
$$

where $C(s):=\Theta_{o} B^{-1} M$ is stable and minimum phase. Consequently

$$
J^{*}=\inf _{Q \in \mathbb{R} \mathcal{H}}\left\|\left(I+\Theta_{i}\left(\Theta_{o} B^{-1} Y-C Q\right) \tilde{M}\right) \frac{\nu}{s}\right\|_{2}^{2} .
$$


By premultiplying $\left[\begin{array}{c}\tilde{\Theta_{i}} \\ I-\Theta_{i}^{-} \Theta_{i}^{-}\end{array}\right]$, we obtain

$$
\begin{aligned}
J^{*}= & \inf _{Q \in \mathbb{R} \mathcal{H}_{\infty}}\left\|\left[\begin{array}{c}
\tilde{\Theta_{i}}+\left(\Theta_{o} B^{-1} Y-C Q\right) \tilde{M} \\
I-\Theta_{i} \tilde{\Theta_{i}}
\end{array}\right] \frac{\nu}{s}\right\|_{2}^{2} \\
= & \left\|\left(\tilde{\Theta_{i}}-\tilde{\Theta_{i}}(0)\right) \frac{\nu}{s}\right\|_{2}^{2}+\left\|\left(I-\Theta_{i} \tilde{\Theta_{i}}\right) \frac{\nu}{s}\right\|_{2}^{2} \\
& +\inf _{Q \in \mathbb{R}_{\infty} \mathcal{H}_{\infty}}\left\|\left(\tilde{\Theta_{i}}(0)+\left(\Theta_{o} B^{-1} Y-C Q\right) \tilde{M}\right) \frac{\nu}{s}\right\|_{2}^{2} \\
= & J_{s}^{*}+\inf _{Q \in \mathbb{R} \mathcal{H}_{\infty}}\left\|\left(\tilde{\Theta_{i}}(0)+\left(\Theta_{o} B^{-1} Y-C Q\right) \tilde{M}\right) \frac{\nu}{s}\right\|_{2}^{2} .
\end{aligned}
$$

It then remains to show (20). For this purpose, define $g(s):=\tilde{M}(s) \nu$. Since $g\left(p_{i}\right)=0$ for any $i \in \mathcal{J}$, we can factorize it as $g(s)=$ $g_{m}(s) \alpha(s)$, where $g_{m}(s)$ is the minimum phase part and has a left inverse analytic in $\mathbb{C}_{+}$[3] , and $\alpha(s)$ is a scalar allpass factor defined by $\alpha(s):=\prod_{i \in \mathcal{J}}\left(\bar{p}_{i} / p_{i}\right)\left(\left(p_{i}-s\right) /\left(\bar{p}_{i}+s\right)\right)$. Consequently

$$
J_{u}^{*}=\inf _{Q \in \mathbb{R}_{\infty} \mathcal{H}_{\infty}}\left\|\left(\frac{\tilde{\Theta_{i}}(0)+\Theta_{o} B^{-1} Y \tilde{M}}{\alpha} \nu-C Q g_{m}\right) \frac{1}{s}\right\|_{2}^{2} .
$$

Now, as in [3], by following a partial-fraction expansion procedure, it then gives rise to

$$
\begin{aligned}
J_{u}^{*}=\inf _{Q \in \mathbb{R} \mathcal{H}_{\infty}} \|\left(\sum_{i \in \mathcal{J}}\left(\frac{p_{i}}{\bar{p}_{i}} \frac{\bar{p}_{i}+s}{p_{i}-s} \frac{1-\tilde{\Theta_{i}}\left(p_{i}\right) \Theta_{i}(0)}{\alpha_{i}}\right)\right. \\
\left.+R-C Q g_{m}\right) \frac{1}{s} \|_{2}^{2}
\end{aligned}
$$

where $R \in \mathbb{R} \mathcal{H}_{\infty}$. By properly selecting $Q \in \mathbb{R} \mathcal{H}_{\infty}$, we obtain

$$
\begin{aligned}
J_{u}^{*} & =\left\|\sum_{i \in \mathcal{J}}\left(\left(\frac{p_{i}}{\bar{p}_{i}} \frac{\bar{p}_{i}+s}{p_{i}-s}-1\right) \frac{1-\tilde{\Theta_{i}}\left(p_{i}\right) \Theta_{i}(0)}{\alpha_{i}}\right) \frac{1}{s}\right\|_{2}^{2} \\
& =\left\|\sum_{i \in \mathcal{J}}\left(\frac{2 \operatorname{Re}\left(p_{i}\right)\left(1-\tilde{\Theta_{i}}\left(p_{i}\right) \Theta_{i}(0)\right)}{\bar{p}_{i} \alpha_{i}} \frac{1}{p_{i}-s}\right)\right\|_{2}^{2} .
\end{aligned}
$$

The result then follows by realizing that

$$
\begin{aligned}
\left\langle\frac{1}{p_{i}-s}, \frac{1}{p_{j}-s}\right\rangle & =\frac{1}{2 \pi} \int_{-\infty}^{\infty} \frac{d \omega}{\left(\bar{p}_{i}+j \omega\right)\left(p_{j}-j \omega\right)} \\
& =\frac{1}{\bar{p}_{i}+p_{j}} .
\end{aligned}
$$

Since $J_{u}^{*}$ is nonnegative, the unstable poles in the plant further worsen the tracking performance, but in a rather complicated way. First, an unstable pole will not affect the tracking performance unless the direction of this pole coincides with the direction of the input signal, namely $\mathcal{R}(P(0))$. Second, unlike in the case of right-invertible MIMO systems, here unstable poles may affect the tracking performance even for minimum phase plants as long as the direction of the plant varies with frequency; in contrast, for right-invertible plants, unstable poles will have an effect only when the plant is nonminimum phase.

\section{ILluSTRATIVE EXAMPLE}

We now illustrate our results with an example. Consider the plant

$$
P(s)=\left[\begin{array}{c}
P_{1}(s) \\
P_{2}(s) \\
P_{3}(s)
\end{array}\right]=\left[\begin{array}{c}
\left(s-z_{1}\right)(s+2) \\
s\left(s+z_{2}\right) \\
s
\end{array}\right] \frac{1}{(s+1)^{3}} .
$$

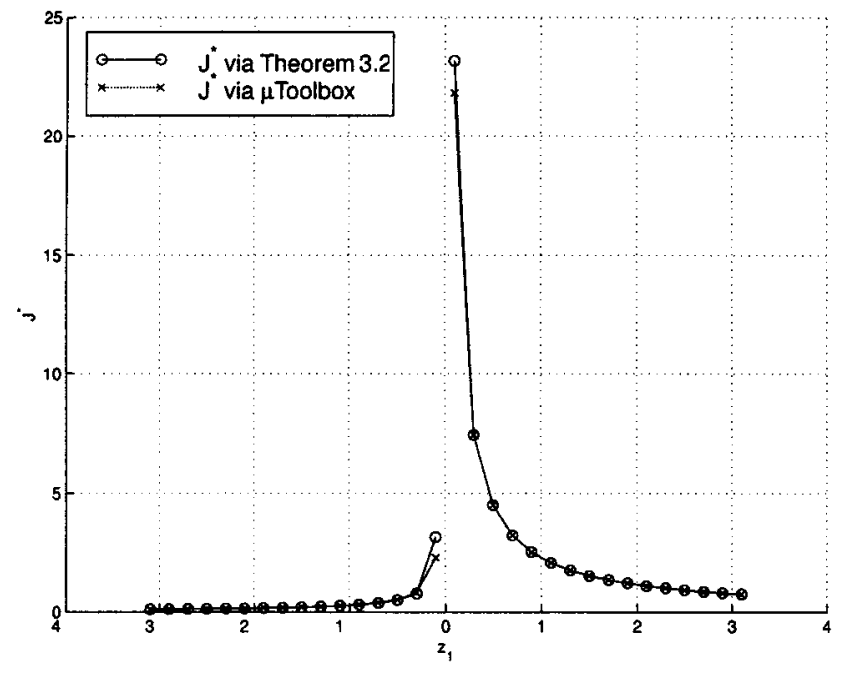

(a)

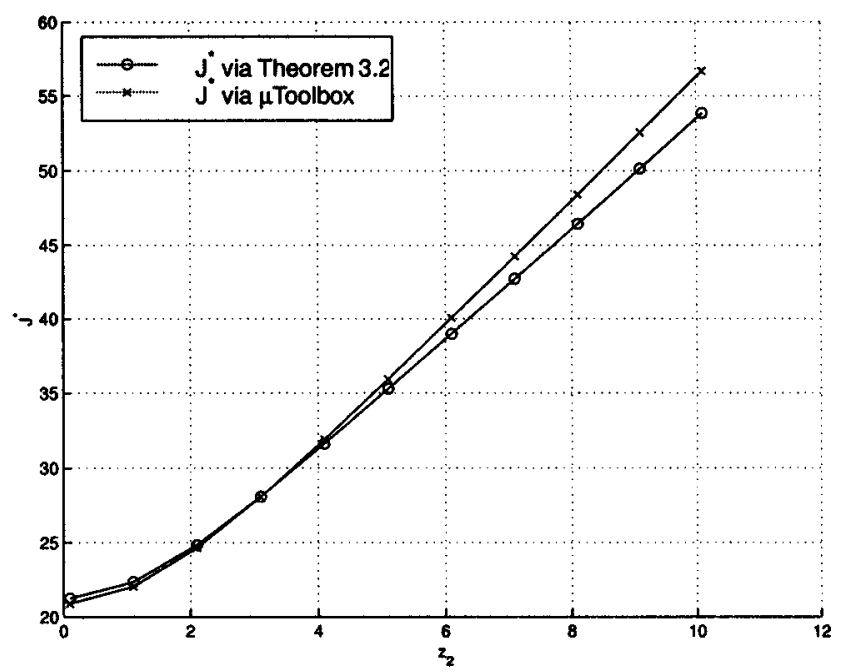

(b)

Fig. 2. (a) $J^{*}$ with respect to $z_{1}$. (b) $J^{*}$ with respect to $z_{2}$.

Let the direction $\nu$ of the step reference input be $[1,0,0]^{T}$ such that Assumption 3.2 is satisfied. We first fix $z_{2}=1$, and let $z_{1}$ vary from -3 to -0.1 and from 0.1 to 3 , subsequently. It is clear that $P_{1}(s)$ possesses a nonminimum phase zero when $z_{1}>0$. For this plant, Fig. 2(a) shows the minimal tracking errors obtained via Theorem 3.2 and that by solving the corresponding $\mathcal{H}_{2}$ optimal control problem with the cost (8) using the MATLAB $\mu$ Toolbox. These two results match rather well. Notably, the plant $P(s)$ yields a worse tracking performance in the presence of the nonminimum phase zero, especially when the zero is closer to the origin. It is also worth pointing out that for the small $z_{2}$, the direction of the plant does not vary much in the low frequency range. Consequently, it can be seen that the effect of the nonminimum phase zero dominates.

Next, we fix $z_{1}=0.1$ and then vary $z_{2}$ from 0.1 to 10 . It is straightforward to verify that for a larger $z_{2}$, the direction of the plant changes more in the low frequency range. Computation results for $J^{*}$ are plotted in Fig. 2(b), which shows that it increases with $z_{2}$. Thus, the results agree to the previous conclusion that the variation of the plant direction with frequency contributes to the tracking difficulty in SIMO systems. Furthermore, it can be seen that this effect can be rather significant. Note that the computational result via the $\mu$ Toolbox yields a more conservative tracking error for it leads to only a suboptimal controller. 


\section{CONCLUSION}

In this note, we have examined the optimal tracking problem for linear time-invariant SIMO systems. Our results characterize analytically how the nonminimum phase zeros, the unstable poles, and more importantly, the plant structure may inherently constrain the optimal tracking performance. Specifically, it is shown that the variation of the plant direction with frequency has a close pertinence on the tracking error. Generally, the more it changes with frequency, the more difficult it is to track the reference input. This is especially so for plants whose direction changes rapidly in the low frequency range.

It is useful to note that our results herein can be extended readily to two-parameter control systems, in a manner similar to [12]. Similarly, in the two-parameter control setting, the plant unstable poles will no longer affect the tracking performance. Moreover, analogous results can be derived in parallel for energy regulation problems associated with multiple-input-single-output plants.

\section{REFERENCES}

[1] G. Chen, J. Chen, and R. Middleton, "Optimal tracking performance for SIMO systems," in Proc. 40th IEEE Conf. Decision Control, Orlando, FL, Dec. 2001, pp. 4869-4874.

[2] J. Chen, "Sensitivity integral relations and design tradeoffs in linear multivariable feedback systems," IEEE Trans. Automat. Contr., vol. 40, pp. 1700-1716, Oct. 1995.

[3] J. Chen, L. Qiu, and O. Toker, "Limitations on maximal tracking accuracy," IEEE Trans. Automat. Contr., vol. 45, pp. 326-331, Feb. 2000.

[4] J. Chen, G. Chen, Z. Ren, and L. Qiu, "Extended argument principle and integral design constraints, Part I: A unified formula for classical results," in Proc. 39th IEEE Conf. Decision Control, Sydney, Australia, Dec. 2000, pp. 4984-4989.

[5] B. A. Francis, A Course in $\mathcal{H}_{\infty}$ Control Theory. Berlin, Germany: Springer-Verlag, 1987, vol. 88, Lecture Notes in Control and Information Sciences.

[6] J. S. Freudenberg, J. W. Grizzle, and B. A. Rashap, "A feedback limitation of decentralized controllers for TITO systems, with application to a reactive ion etching," in Proc. 33th IEEE Conf. Decision Control, Orlando, FL, Dec. 1994, pp. 2312-2317.

[7] J. S. Freudenberg and R. H. Middleton, "Properties of single input, two output feedback systems," Int. J. Control, vol. 72, no. 16, pp. 1446-1465, 1999.

[8] — " "Feedback systems with an almost rank deficient plant," in Proc. IEEE Amer. Control Conf., San Diego, CA, 1999, pp. 409-413.

[9] M. Morari and E. Zafiriou, Robust Process Control. Upper Saddle River, NJ: Prentice-Hall, 1989.

[10] N. Morse, R. Smith, B. Paden, and J. Antaki, "Position sensed and selfsensing magnetic bearing configurations and associated robustness limitations," in Proc. of 37th IEEE Conf. Decision Control, Tampa, FL, Dec. 1998, pp. 2599-2604.

[11] L. Qiu and E. J. Davison, "Performance limitations of nonminimum phase systems in the servomechanism problem," Automatica, vol. 29, no. 2, pp. 337-349, 1993.

[12] L. Qiu and J. Chen, "Time domain performance limitations of feedback control," Math. Theory Networks Syst., pp. 369-372, 1998.

[13] M. M. Seron, J. H. Braslavsky, and G. C. Goodwin, Fundamental Limitations in Filtering and Control. London, U.K.: Springer-Verlag, 1997.

[14] A. R. Woodyatt, M. M. Seron, J. S. Freudenberg, and R. H. Middleton, "Cheap control tracking performance for nonright-invertible systems," in Proc. 37th IEEE Conf. Decision Control, Tampa, FL, Dec. 1998, pp. 4362-4367.

[15] A. R. Woodyatt, "Feedback control of multivariable nonsquare systems," Ph.D. dissertation, Dept. Elect. Comput. Eng., Univ. Newcastle, Newcastle, Australia, Jan. 1999.

\section{A New LMI Condition for Robust Stability of Polynomial Matrix Polytopes}

\author{
Paulo J. de Oliveira, Ricardo C. L. F. Oliveira, and Pedro L. D. Peres
}

\begin{abstract}
A sufficient condition for checking the robust stability of a polytope of polynomial matrices is proposed in this note. A simple feasibility test performed in a convex set of linear-matrix inequalities defined at the vertices of the polytope yields sufficient conditions for the robust stability of the entire domain. Both continuous-time (left half-plane) and discrete-time stability (unit disk) are investigated. Numerical comparisons with quadratic stability results and with another method recently appeared in the literature show that the conditions proposed provide, in general, less conservative results.
\end{abstract}

Index Terms-Linear-matrix inequalities (LMIs), parameter dependent Lyapunov functions, polynomial matrices, robust stability, uncertain polynomials.

\section{INTRODUCTION}

Polynomial matrices are an important tool for control systems, being capable of representing more naturally the system dynamics in many cases [1]. Several methods for analysis and control are based in polynomial matrices, and it is well-known that the denominator polynomial matrix in a matrix fraction description possesses useful information about stability and performance of the system [2], [3].

When uncertain parameters affect the system, the analysis of robust stability requires the verification of the stability of uncertain polynomial matrices, and if polytopic uncertainty is considered, the polytope of polynomial matrices need to be checked. In fact, polytopic domains are a quite general representation of parameter uncertainty [4]. During the last two decades, several papers dealing with robust control of linear systems in convex bounded (polytopic) domains have appeared, in both state space and polynomial representation. One of most useful techniques for dealing with uncertainty is without doubt the Lyapunov-based approach (see, for instance, [4]-[6]). For a discussion about polynomial matrices and related numerical methods for stability tests, see [7].

As a matter of fact, the robust stability of polynomials has been largely addressed in the last years, as in the very known result of Kharitonov for testing polynomials with coefficients inside an interval and in the edge theorem [4], [5]. The extension of these results to the case of polynomial matrices does not seem to be immediate, and it has been proven that checking stability of multilinear polynomial families is an $N P$-hard problem [8].

In [7], linear matrix inequality (LMI) conditions, which had recently appeared in the literature for testing the robust stability of a polytope of matrices [9], [10], have been extended to the case of polynomial matrix polytopes. More recently, new LMI conditions which turn to be less conservative have been provided for checking the robust stability of continuous-time [11] and discrete-time [12] uncertain systems.

In this note, sufficient conditions for the robust stability of polynomial matrix polytopes are given in terms of LMIs, which are obtained as

Manuscript received March 11, 2002; revised May 13, 2002. Recommended by Associate Editor P. Apkarian. This work was supported in part by the Brazilian agencies CAPES and CNPq.

P. J. de Oliveira is with DCET-UNEB, 41195-000 Salvador BA, Brazil (e-mail: james@dt.fee.unicamp.br).

R. C. L. F. Oliveira and P. L. D. Peres are with the School of Electrical and Computer Engineering, University of Campinas, 13081-970 Campinas SP, Brazil (e-mail: peres@dt.fee.unicamp.br).

Digital Object Identifier 10.1109/TAC.2002.803560. 